\title{
Investigating lymph - a new challenge and perspective in breast cancer
}

\author{
Georgiana Constantin ${ }^{1,2}$, Cristian Bordea ${ }^{3,4}$, Alexandra Totan', \\ Iulia-Ioana Stanescu-Spinu', Daniela Miricescu' ${ }^{1}$, Maria Greabu ${ }^{1}$ \\ ${ }^{1}$ Department of Biochemistry, Faculty of Dental Medicine, "Carol Davila“ University of Medicine and \\ Pharmacy, Bucharest, Romania \\ ${ }^{2}$ Special analysis laboratory for liver transplantation, Fundeni Clinical Institute, Bucharest, Romania \\ ${ }^{3}$ Department 10 Surgery, Discipline of Surgical Oncology, Faculty of Medicine, \\ "Carol Davila" University of Medicine and Pharmacy, Bucharest, Romania \\ ${ }^{4}$ "Prof. Dr. Alexandru Trestioreanu“ Oncology Institute, Bucharest, Romania
}

\begin{abstract}
Worldwide breast cancer $(B C)$ represents one of the most common type of cancer. Mortality from this disease is declining due to new therapies, but many patients face the side effects of cancer and treatment. The most common side effect is the lymphedema of the upper extremity. Although the incidence of lymphedema secondary to breast cancer is $63.4 \%$, there are few studies on the immune mechanisms responsible for lymphedema and healing. Extensive research of the development, structure and function of the lymphatic system must be performed to identify the role of lymphatic vessels in breast cancer. All this can lead to discovery of new potential biomarkers in lymph in order to improve early detection and treatment of lymphedema and the current strategies for controlling tumor metastasis.
\end{abstract}

Keywords: breast cancer, lymphedema, inflammation, cytokines, growth factors

\section{INTRODUCTION}

Worldwide, breast cancer (BC) is the most frequently diagnosed type of cancer among women. Around the globe, in 2020, there were 2.3 million new cases of breast cancer and 685000 deaths. Breast cancer incidence is increasing in every state, in women of every age, after puberty, but most common in later life. After lung cancer, breast cancer is on top of the causes of death (1).

Because breast cancer is a major public health issue, new and accurate methods for early screening and treatment need to be developed and applied (2). In the last years, the serum concentrations of tumor markers have been used to detect tumor activity (3). Unfortunately, the real usefulness of tumor markers has been questioned because of the low sensitivity for early disease (4).

Effective treatments depend of the stage and type of breast cancer and of how far it has spread.
Fortunately, there are several types of treatments: surgery, chemotherapy, hormonal therapy, biological therapy, radiation therapy that have saved lives, with survival from this type of tumor increasing by $1.4 \%$ annually (5). Unfortunately, many of these women face challenges in their activities of daily living, as a result of both the illness and the treatments that have saved their lives. One of the most widely studied treatment-related adverse effect is lymphedema of the upper extremity. It is estimated that more than one out of every five women treated for BC will develop lymphedema $(20 \%$ in 6 months, $36 \%$ in 1 year and $54 \%$ within 36 months). The most relevant risk factors are the number of lymph nodes excised in surgery, radiation therapy, chemotherapy, infection and obesity (6). This disruption can cause a backup of lymph fluid in the interstitial area, causing swelling, which leads to physical discomfort like pain, heaviness, tightness, numbness, fatigue, and stiffness in 
the affected limb. Also, these symptoms lead to a decreased physical and psychological function and social well-being in women with lymphedema (7).

Therefore, research in this area is necessary in order to prevent such unintended adverse effect for breast cancer survivors and to understand the development and function of the lymphatic system and the diseases related to it.

An interesting, yet understudied aspect is the fact that postoperatively, the amount of drained lymph and healing time differ from patient to patient. Extensive research into the development, structure and function of the lymphatic system must be performed to identify the role of lymphatic vessels in disease processes and its role in immune function (8). All this can lead to the discovery of new potential biomarkers in lymph and serum in order to improve early detection and treatment of lymphedema.

\section{THE LYMPHATIC SYSTEM}

The lymphatic system is a major part of the circulatory and immune system, present in all of the vascularized organs, except retina, cartilages and brain. It consists of primary lymphoid organs: bone marrow and thymus and of secondary lymphoid organs: lymphatic vessels, lymph nodes, spleen, adenoids, Peyer's patches, appendix and lymphoid tissue (9).

Through a filtration process, the huge linear network of lymphatic vessels collects extravasated fluid, cells, proteins and lipids, which form the lymph. They are drained into the initial lymphatic capillary vessels, situated at the interstitial space of every parenchymal organ. The lymphatic capillaries are blind-ended tubes with one layer of lymphatic endothelial cells (LEC), which enable the fluid entry into the vessels through discontinuous button-like junctions (10). Then, the lymph is transported to thicker collecting lymphatic capillaries which are connected with many lymph nodes disseminated throughout the body (11). The collecting lymphatics are complex vessels with continuous intracellular zipper-like junctions, basement membrane and a contractile smooth muscle layer (12).

In lymph nodes bigger than $100 \mathrm{kDa}$, molecules are captured or transported to the next lymph node through the efferent lymphatic vessel by unidirectional transport (13). Finally, they return to blood circulation via the thoracic duct to the subclavian vein (13).

Because lymph is an exudate from blood vessels, formed as a result of capillary filtration, it was considered to be a plasma ultrafiltrate rich in lymphocytes, macrophages and plasma proteins. But recently, proteomic analyses revealed several tissue specific proteins in lymph different from plasma composition (13).

Therefore, besides the well-known role in regulation the tissue pressure and fluid homeostasis, by uptaking fluids and plasma solutes from the interstitial fluid (IF) and returning back to the venous circulation, lymphatics also play an important role in immune surveillance by trafficking lymphocytes and antigen presenting cells (APC) to regional lymph nodes, where specific immune responses are initiated. Also, the lymphatic system contributes to chronic inflammation and is associated with autoimmune diseases (psoriasis, rheumatoid arthritis) and many disease processes, including cancer progression or lymphedema. Hence, we need to identify specific lymph markers related to $\mathrm{BC}$ and other diseases, that can help us improve the current strategies for controlling tumor metastasis and some undesirable and often encountered side effect of cancer treatment, such as lymphedema, taking in consideration that after a mastectomy, up to $49 \%$ develop upper limb lymphedema (13).

\section{INFLAMMATION AND CANCER: A BIDIRECTIONAL RELATIONSHIP}

Inflammation and cancer are linked in a twoway relationship. Tumors induce inflammatory microenvironment and host immune response; on the other hand, chronic inflammation via proinflammatory cytokines and reactive oxygen species (ROS) induces: initiation, progression and metastasis of cancer cells (14). BC initiation and dissemination is triggered by immune cells and inflammatory biomarkers in tumor microenvironment. The balance between antitumoral markers and local inflammatory markers contributes to the progress or elimination of the tumor. Inflammation induced in the process of tumor progression is one of the reasons why the immune system cannot block BC development (15). Inflammation is also one of the main secondary effects after BC treatment (Figure 1). Consistent association of inflammation has been correlated with $\mathrm{BC}$ treatment resistance, metastasis or even cancer relapses. Therefore, patients who received anti-inflammatory plus antitumoral treatment had a more promising outcome (15).

Thus, inflammatory cells operate in conflicting ways: both tumor-antagonizing and tumor-pro- 


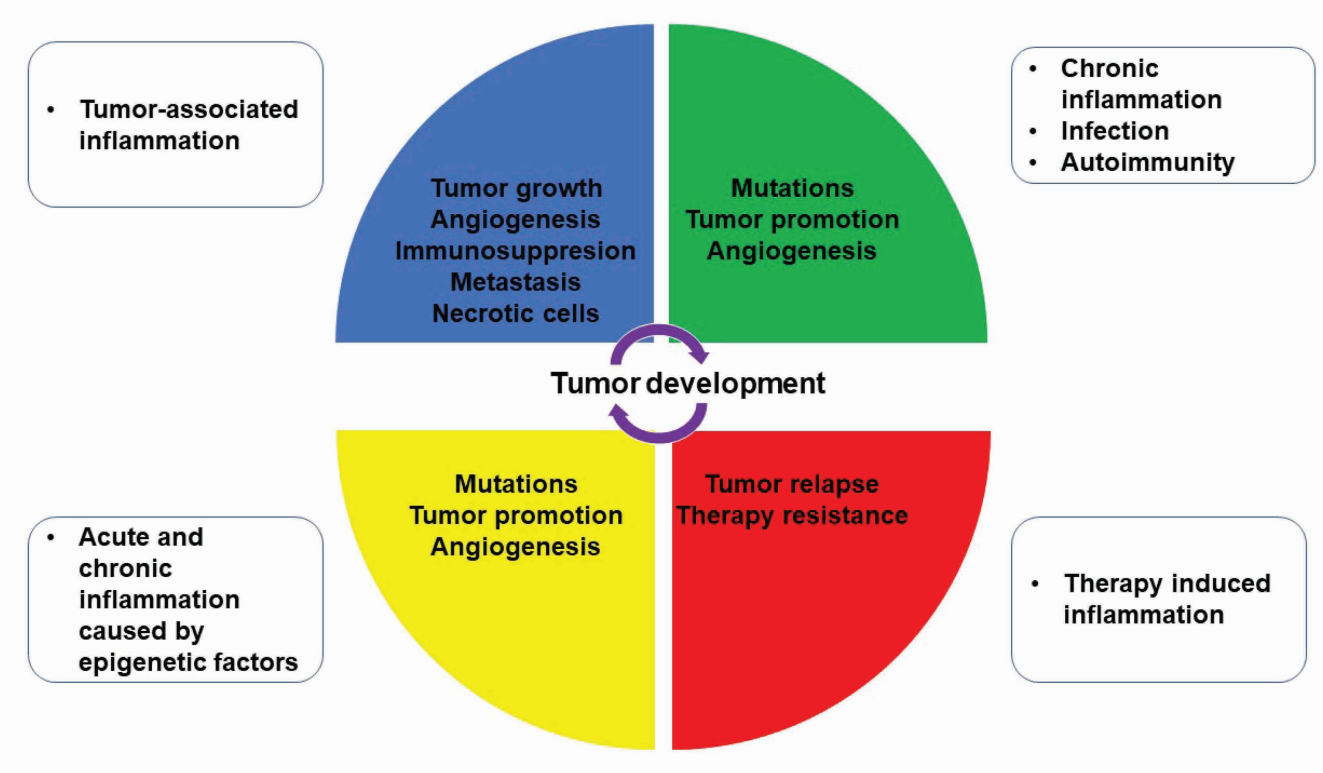

FIGURE 1. Types of inflammation in cancer

moting. Some of tumor-promoting inflammatory cells are macrophage cells, mast cells, neutrophils, $\mathrm{T}$ and $\mathrm{B}$ lymphocytes (16).

These inflammatory cells release signaling molecules that serve as effectors of their tumor-promoting actions, such as epidermal growth factor (EGF), the angiogenic vascular endothelial growth factor (VEGF), fibroblast growth factor 2 (FGF2), chemokines and cytokines that amplify the inflammatory state (16). Also, they produce proangiogenic and/or pro-invasive matrix-degrading enzymes, for example matrix metalloproteinases (MMPs), cysteine cathepsin proteases, and heparanase (17). As a result of these diverse effectors, inflammatory cells induce angiogenesis, cancer cell proliferation and metastatic dissemination $(17,18)$.

Other cells with proinflammatory potential that are worth mentioning are necrotic cells. Unlike apoptosis cells, they release proinflammatory signals into the tumor microenvironment, recruiting more and more inflammatory cells of the immune system (16), with the role of removing necrotic debris. But in the BC context, immune inflammatory cells become tumor promoting cells. Moreover, necrotic cells release regulatory factors, for example interleukin-1 $\alpha$ (IL-1 $\alpha$ ), which stimulates tumor cells' proliferation. Finally, necrotic cells may do more damage in $\mathrm{BC}$, by bringing growth-stimulating factors to the tumor environment (16).

Inflammation and immunity are also regulated by another category of signaling molecules named cytokines. They play an important role in the development of chronic inflammation, autoimmunity and cancer. Lately, the cytokine storm has been mentioned in relation with the COVID-19 pandemic. The term cytokine storm is used to describe a massive discharge of proinflammatory cytokines induced in a dysregulated manner in response to infection, trauma, therapy, chimeric antigen-receptor T-cell (19). Inflammation is an intricate process in which multiple cells (B lymphocytes, T lymphocytes, epithelial cells, fibroblast, endothelial cells, myeloid cells, muscle cells, adipocytes) interact with each other through membrane-associated receptors, MMPs and soluble factors, such as cytokines: for example, interleukin-1 $\beta$ (IL-1 $\beta$ ), tumor necrosis factor alpha (TNF- $\alpha$ ), interleukin-6 (IL-6), interleukin-17 (IL-17), chemokines and growth factors (19). IL-6 is an important marker related to chronic inflammation, autoimmunity, cancer and cytokine storm $(20,21)$.

Chronic inflammation is linked bidirectionally with autoimmune diseases and cancer (22). Almost $10 \%$ of cancers are initiated by activation of inherited gene defects, and $90 \%$ are a result of environmental factors (infection, stress, obesity, aging, smoking), which can induce chronic inflammation (23). Important molecules implicated in this response are immune cells (macrophages, $\mathrm{T}$ cells, dendritic cells) and non-immune cell ( endothelial cells, adipocytes, fibroblasts, epithelial cells, tumor cells, tumor microenvironment), cytokine IL$1 \beta$, TNF- $\alpha$, IL- 6 , transcription factors nuclear factor-kappa B (NF-kB), signal transducer and activator of transcription 3 (STAT3) (19). Studies have shown a connection between inflammation and oncogenesis via IL-6-STAT3 signaling through 


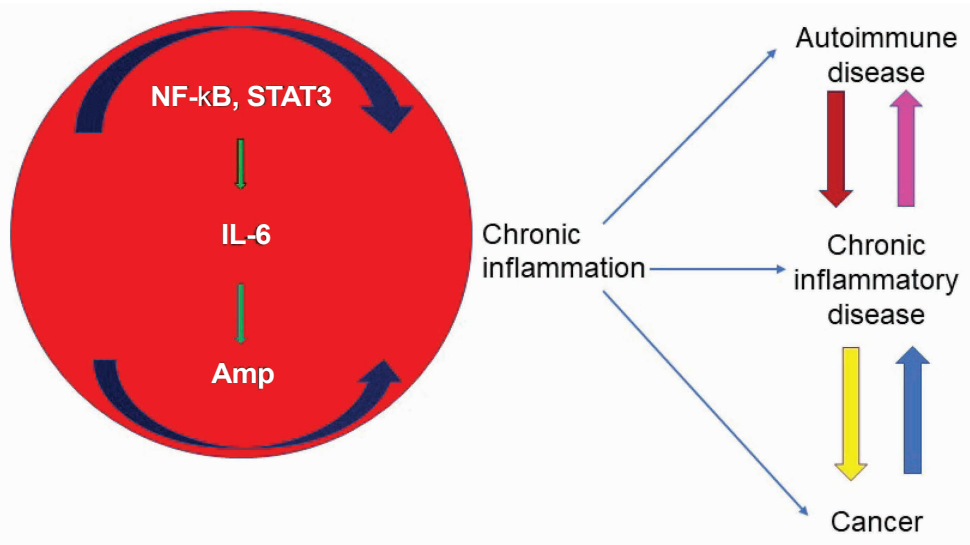

FIGURE 2. IL-6 activation. Activating both STAT3 and NF-KB leads to activation of IL-6 Amp in non-immune cells. As a result, IL-6 Amp hyperstimulates the production of IL-6, other cytokines and growth factors. They also activate other immune cells, leading to further activation of IL-6 Amp with an uncontrolled inflammatory response linked to autoimmune diseases, chronic inflammatory diseases and cancer synergistic effect of STAT3 and NF-кB (24). The target of hyperactivated NF- $\kappa \mathrm{B}$ is IL- 6 through the positive feedback loop called IL-6 amplifier (IL-6 Amp) (Figure 2). Therefore, STAT3 and NF- $\kappa$ B play an important role in inflammatory diseases and $\mathrm{BC}$ development through synergistic activation of IL-6 (25).

\section{POTENTIAL BIOMARKERS DETECTED IN SERUM AND LYMPH FOR BREAST CANCER INVESTIGATION}

The lymph is an extracellular fluid, with unique components, such as products of tissue metabolism and catabolism, immune cells, derived from most adjacent parenchymal organs, since the lymph is in direct contact with each cellular layer of the parenchymal interstitial space to its collection into the lymphatic vessels (26).

Its composition differs depending on the physiological or pathological processes in the tissue, such as acute or chronic inflammation, trauma, cancer. The main physiological role is to ultrafiltrate fluid from blood capillaries into interstitial space of the adjacent tissue, and to return it to blood circulation. That is why most of the proteins found in the blood are also present in the interstitial fluid. Any disturbance of this physiological function will result in lymphedema. The main cause of this condition is the accumulation of protein-rich fluid in the adjacent tissue. In addition to lymphedema, the process of lymphangiogenesis is also found in other pathologies, such as acute and chronic inflammation and cancer. Therefore, recent studies focus on the analysis of lymph and the detection of protein biomarkers in serum and lymph specific to breast cancer and other pathologies. Although early studies have confirmed that the lymph is rich in common proteins with plasma and blood (27), in the last 15 years of lymph inves- tigations a proteome more unique and complex than in the plasma has been discovered. According to International Immunology Online, 1063 lymph proteins have been discovered, including $630 \mathrm{hu}-$ man lymph proteins. Other studies have published a comparative analysis on paired lymph and plasma samples and observed the presence in the lymph of 72 proteins with a role in apoptosis, cell catabolism and proteins belonging to the extracellular matrix $(28,29)$.

In plasma versus lymph samples from patients who suffered major trauma, 105 lymph-specific immunomodulatory products were identified with proinflammatory role, neoangiogenesis and cell lysis, while 232 were shared features (30).

Therefore, due to the quantitative and qualitative differences between the two biological fluids, lymph analysis should be continued in order to discover specific proteins drained in the lymph nodes with the role in inflammation and cancer. Endothelial cells are part of the tumor microenvironment, on which tumor cells act by secreting growth factors that cause the formation of new lymphatic vessels, with a role in tumor progression and metastasis. Lymphatic vessels also carry antigens and antigen-presenting cells to the lymph nodes to activate an antitumor immune response. As a result, dysfunctional lymphatic vessels can lead to unproper response of the immune system (31).

Distinct biomarkers for lymphatic endothelial vessels and not for blood endothelial cells, have been identified: for example, podoplanin (PDPN), vascular endothelial growth factor receptor-3 (VEGFR-3), prospero homebox protein (PROX1), lymphatic vessel hyaluronan receptor-1 (LYVE1), lymphatic-specific chemokine CCL21, neuropilin-2, a co-receptor for vascular endothelial growth factor C (VEGF-C) and hepatocyte growth factor (HGF) (9). 
In vitro studies showed that high levels of LYVE1, PDNP, PROX1, and VEGFR-3 were detected in the lymph vessel of BC patients and also revealed a corelation with the development of lymphatic metastasis (32).

\section{Podoplanin (PDPN)}

Podoplanin is a specific lymphatic endothelium biomarker, a transmembrane sialoglycoprotein synthesized under the action of the PDPN gene and is expressed by the lymphatic endothelial cells (LECs), but not in blood vascular endothelial cells in vivo and in vitro (33), with the main role in separating the lymphatic system from the circulatory one, through the development process of vertebrates. This process is possible by ortho-glycosylation of podoplanin on endothelial lymphatic cells due its interaction with platelets and lectins, which make possible platelet aggregation and adhesion (9). Also, we might speculate another role of podoplanin in preventing anastomoses between lymphatic vessels and blood vessels through thrombus formation. Through the activation of platelets, the proliferation of lymphatic vessels is inhibited and thus, separation from the blood system takes place. During embryonic development, the circulatory system undergoes a constant remodeling process depending on the changes that take place in the adjacent tissues. Through proper functioning of endothelial PDPN, takes place the distinct development of the lymphatic system from the blood system (34). One of the PDPN receptors that binds to activated platelets is the C-type-lectin-like receptor 2, also expressed in other blood cells and involved in lymphangiogenesis and cancer (35).

The process of lymphangiogenesis that takes place in the tumor microenvironment and adjacent lymph nodes is correlated with cancer progression through invasion, metastasis and poor prognosis (36). Malignant tumors initially metastasize to the lymph nodes and then spread throughout the body through the lymphatic system.

Podoplanin is also expressed by cancer-associated fibroblast, epithelial cells and endothelial cells, where it stimulates cellular motility and reduces cellular adhesion. Podoplanin expression by fibroblasts in the tumor microenvironment of breast cancer correlates with tumor aggression factors: lymphovascular invasion, lymph node metastasis, poor prognosis (37).

\section{Vascular endothelial growth factor (VEGF) family}

The main signaling pathway responsible for lymphangiogenesis is the VEGF- receptor
(VEGF-R) family. By binding VEGF-C and VEGF-D to their receptors VEGFR-2 and VEGFR-3, new lymphatic vessels are formed and maintained in adulthood (8).

Numerous studies have investigated the potential of VEGF-C to induce lymphangiogenesis. Unfortunately, the treatment of lymphedema by stimulating VEGF-C alone is not enough, because mediators are also needed to stabilize the lymphatic vessels. The development of endothelial cells from the embryonic veins takes place through signals transmitted by the PROX1 and VEGF-C pathways. Activation of VEGF-C leads to the expression of PROX1 in endothelial cells for migration and lymph sacs formation in the embryo (38). After the formation of the lymphatic sac, the lymphatic system and the blood system develop separately, the only connections between them allow the return of lymph to the blood system (38).

Along with VEGF-C, VEGF-D plays an important role in the development of LECs (lymphatic endothelial cells). The action of VEGF-C and VEGF-D on endothelial cells occurs by binding to specific tyrosine kinase receptors on their surface, namely VEGFR-2 and VEGFR-3 (9). VEGFR-2 is responsible for the formation of new blood vessels by angiogenesis and VEGFR-3 on LECs is responsible for the growth of lymphatic vessels by lymphangiogenesis, therefore is a specific marker exclusively expressed on the lymphatic endothelium (39).

Hence, VEGF-C and its receptor VEGFR-3 are indispensable for early lymphatic development, while the lack of VEGF-D -the only other ligand of VEGFR 3, does not affect lymphatic vessels development (40). However, after proteolytic cleavage, both VEGF-C and VEGF-D can bind to VEGFR-2, whose role in lymphangiogenesis has not yet been elucidated (40).

Activation of the VEGF-C / VEGF-D / VEGFR-3 signaling pathway increases the permeability of lymph vessels, leading to increased flow to the lymph nodes with tumor cell invasion and lymph metastasis (12).

Another growth factor from the family of VEGF is VEGF-A, that binds on VEGFR-2 and not VEGFR-3 and has lymphangiogenic potential. VEGF-A also plays an important role in inflammation associated with lymphangiogenesis; thus, it could block the inflammatory cascade of the lymph node by activating anti-VEGF-A antibodies. Studies show that inflammation associated with VEGF-Ainduced lymphangiogenesis worsens the inflammatory process (41). 
The process of lymphangiogenesis in adults takes place only in pathological conditions: post-traumatic, inflammatory diseases or tumor growth. Primary metastasis of solid tumors occurs most frequently through the lymphatic system. Tumor cells can spread to the entire body through the affected lymph nodes by lymphatic remodeling. Studies show that overexpression of VEGF-C and VEGF-D stimulates the growth of tumor peripheral lymph vessels by upregulating cyclooxigenase-2 (COX2) and prostaglandin E2 production by macrophages (42). Since lymphangiogenesis is influenced by the action of prostaglandins, the use of nonsteroidal anti-inflammatory drugs (NSAIDs) in the treatment of metastasis may be attempted.

More recently, it has been discovered that tumors can act remotely for future metastasis of a distant organ by initiating a premetastatic niche in the organ to be disseminated. For example, in experiments on mice, in the lymph nodes but also in the lungs, a metastatic niche was formed by the action of VEGF-A, which stimulated angiogenesis and vascular permeability, and by activating C-C Motif Chemokine Ligand 5 (CCL5), a chemotactic factor for tumor cells in response to tumor-derived IL-6 (42).

VEGF-C also stimulates the production of C-C motif ligand 21 (CCL21) chemokines in the lymphatic endothelium surface, thus facilitating the entry of CCR7 positive tumor cells in the initial lymphatic vessel, and then interact with and crawl on LECs as they travel to the lymph node (43).

So, CCR7 and VEGF-C expression by breast cancer cells stimulates lymphovascular invasion. By increasing the pressure in the lymphatic vessels, the tumor cells are redirected to the sentinel lymph node through the autologous secretion of chemokine receptor 7 (CCR7) (44). VEGF-C, secreted by tumor cells and its receptor VEGFR-3, stimulates the expression of CCL21 in endothelial cells. Superactivated CCL21 binds to its CCR7 ligand on tumor cells and facilitates tumor invasion of lymph vessels. This process highlights the ability of tumor cells to use chemokines to self-direct to their preferred organ for metastasis. The chemokine pair CCL21/CCR7 is involved in the proliferation and metastasis of many types of tumors, including breast cancer (44).

CCR7 is a predictive marker for axillary lymph node metastasis in breast cancer. CCR7 is correlated with lymphovascular markers (LYVE1, Podoplanin, Prox-1) and VEGF-C. Overexpression of CCR7 correlates with: primary tumor size, invasiveness, and poor survival rate. In vivo, the meta- static process is decreased, when CCL21 expression is blocked in the secondary lymphoid organs, leading to diminishing the antiapoptotic and chemotactic effect of CCR7 positive tumor cells (45). Immunologically, CCR7 is located on the surface of dendritic cells and $\mathrm{T}$ cells, while its ligand CCL21 is found on the surface of the lymphatic endothelium. Both CCL21 / CCR7 axis and VEGF-C are important players of lymphangiogenesis and tumor cell dissemination. It is worth mentioning that a close loop of communication exists between pairs CCL21 / CCR7 and VEGF-C/VEGFR-3, in a sense that not only VEGF-C promotes CCL21 secretion by LECs, but also CCR7 activation stimulates VEGF-C synthesis by tumor cells and promotes lymphangiogenesis (45).

CCL2 1 also stimulates lymphangiogenesis in a LEC-dependent manner: by stimulating the lymphangiogenic traits of LECs and by modulating the VEGFR-3 signaling pathway. Therefore, CCR7 strongly correlates with VEGF-C expression in breast cancer. Activation of the CCR7 / CCL21 axis stimulates the phosphorylation of the protein kinase B (AKT) pathway and extracellular signal-regulated kinase (ERK1/2) pathway in BC cells, but only the AKT signaling pathway is involved in the regulation of VEGF-C secretion by tumor cells (45).

On BC cells, the expression of CXCR4 and CCR7 chemokine receptors was correlated with lymph node metastasis. Therefore, targeted treatment of these signaling pathways could block tumor cell metastasis to the lymph nodes and could be a potential therapeutic opportunity for lymphedema patients. In patients with breast cancer, the process of lymphangiogenesis in the sentinel lymph node was correlated with distant metastasis of the lymph nodes. Consequently, inhibiting the formation of new lymphatic vessels could prevent cancer metastasis. One possibility is to block the VEGFR-3 signaling pathways, canceling the effects of VEGF-C and VEGF-D, using anti-VEGFR-3 antibodies or other ligands, such as the VEGFR-3-Ig fusion protein or gene silencing through small interfering RNA-mediated VEGF-C (46). In vitro research has shown that by blocking the VEGF-C / -D / VEGFR-3 signaling pathway, an anti-lymphangiogenic effect is produced in the tumor, but it does not produce any action on normal lymphatic vessels. VEGFR-3 in normal tissue is expressed only in the lymphatic endothelium, while in the tumor tissue it is involved in the angiogenesis of blood vessels. Thus, through the action of anti-VEGFR-3 neutralizing antibodies, we obtain an antiangiogenic effect on tumor vessels (46). 
Additionally, VEGF-C and VEGF-D can stimulate the VEGFR-2 / VEGF-A signaling pathway, amplifying metastasis and lymphangiogenesis, so the solution would be to use anti-VEGFR-2 antibodies. Another potential solution is the use of anti-neuropilin-2 antibodies, a non-kinase receptor for VEGF-C, involved in the formation of new lymphatic vessels and metastasis (46).

Tumor cells secrete growth factors that act on the lymph vessels, but the latter also secrete chemokines to attract tumor cells into the lymph vessels. For example, the chemokine CCL21, secreted by lymphatic endothelial cells, binds to the CCR7, a receptor specific to tumor cells and allows the invasion of tumor cells in the regional lymph nodes. Thus, a potential target for inhibiting lymphatic metastasis is to knock-down CCL21, which diminishes the chemotactic effect of CCR7 in secondary lymphoid organs and correlates significantly with down-regulation of VEGF-C (45).

\section{Prospero homebox protein (PROX1) and lymphatic vessel hyaluronan receptor-1 (LYVE-1)}

They are both lymphendothelial precursors capable of integrating into growing lymphatics during the embryonic development of the lymphatic vasculature. PROX-1 expression is restricted to LECs, being known as "the master control gene for lymphatic development" (11).

LYVE-1 is a homolog of the blood vascular endothelium specific hyaluronan receptor CD44. It is expressed on the endothelial cells of the anterior cardinal vein at early stage of development and later restricted in LECs. Although it is one of the most researched endothelial lymphatic markers, it is also expressed in activated tissue macrophages and endothelial cells of liver and spleen, but the function of this protein remains still unknown (40).

Conflict of interest: none declared

Financial support: none declared

\section{REFERENCES}

1. Pusina S. Correlation of serum levels of urokinase activation plasminogen (UPA) and its Inhibitor (PAl-1) with hormonal and HER-2 status in the early invasive breast cancer. Med Arch. 2018; 72(5):335-340.

2. Mansouri H, Mnango LF, Magorosa EP, et al. Ki-67, p53 and BCL-2 expressions and their association with clinical histopathology of breast cancer among women in Tanzania. Sci Rep. 2019;9(1):9918.

3. Ahmed M. Tumor markers of breast cancer: New prospectives. Journal of Oncological Sciences. 2017;3(1):5-11.

4. Marić $P$, Ozretić $P$, Levanat $S$, et al. Tumor markers in breast cancer-evaluation of their clinical usefulness. Coll Antropol. 2011;35(1):241-7.

5. https://www.aecc.es/es/todo-sobre-cancer/tipos-cancer/cancermama. Accessed 13 Mar 2021.

6. Muñoz-Alcaraz MN, Pérula-de-Torres LÁ, Serrano-Merino J, et al. Efficacy and efficiency of a new therapeutic approach based on

\section{Predictive markers of lymphedema secondary to breast cancer}

Genotypic studies in women with lymphedema after breast cancer have identified three haplotypes responsible for this treatment-related adverse effect: forkhead box protein C2 (haplotype A03), neuropilin 2 (haplotype F03) and VEGF-C (haplotype B03) (8). Other potential biomarkers for lymphedema are proteins involved in lymphangiogenesis, inflammation, fibrosis and lipid metabolism: fibroblast growth factor $\beta$, IL-4, IL-10, TNF- $\beta$ and leptin (47). New research is needed on the lymphatic system to treat lymphedema, cancer or inflammatory diseases.

\section{CONCLUSIONSANDFUTURECHALLENGES}

Lymph is an exudate from blood vessels, rich in lymphocytes, macrophages and plasma proteins. However, recent proteomic studies revealed that this ultrafiltrate also contains several specific proteins. In women with breast cancer, one of the most common treatment-related adverse effect is lymphedema of the upper extremity. Our research analyzed the immune mechanisms responsible for lymphedema and healing in these patients. Moreover, our study emphasizes a significant crosstalk displayed by CCR7 and VEGF-C, suggesting a novel role of CCL21/CCR7 in the promotion of breast cancer induced lymphangiogenesis. In future research, it is of utter importance to identify biomarkers that block lymph angiogenesis and metastasis, in order to have a better outcome for breast cancer patients. Genome-wide expression analyses of lymph and LECs could detect molecules involved in the complex molecular processes of lymphatic regulation under pathological conditions for a long-term disease control.

activity-oriented proprioceptive antiedema therapy (TAPA) for edema reduction and improved occupational performance in the rehabilitation of breast cancer-related arm lymphedema in women: a controlled, randomized clinical trial. BMC Cancer. 2020;20(1):1074.

7. Pusic AL, Cemal Y, Albornoz C et al. Quality of life among breast cancer patients with lymphedema: A systematic review of patientreported outcome instruments and outcomes. Journal of Cancer Survivorship. 2013;7(1):83-92.

8. Padera TP, Meijer EF, Munn LL. The lymphatic system in disease processes and cancer progression. Annu Rev Biomed Eng. 2016;18:125-158.

9. Alderfer L, Wei A, Hanjaya-Putra D. Lymphatic tissue engineering and regeneration. J Biol Eng. 2018;12:32.

10. Baluk P, Fuxe J, Hashizume $H$, et al. Functionally specialized junctions between endothelial cells of lymphatic vessels. J Exp Med. 2007;204(10):2349-62. 
11. Choi I, Lee S, Hong YK. The new era of the lymphatic system: no longer secondary to the blood vascular system. Cold Spring Harb Perspect Med. 2012;2(4):a006445.

12. Gutierrez-Miranda L, Yaniv K. Cellular Origins of the Lymphatic Endothelium: Implications for Cancer Lymphangiogenesis. Front Physiol. 2020;11:577584.

13. Sixt M, Kanazawa N, Selg M, et al. The conduit system transports soluble antigens from the afferent lymph to resident dendritic cells in the T cell area of the lymph node. Immunity. 2005;22(1):19-29.

14. Kaur RP, Rubal, Banipal RPS, et al. Association of elevated levels of C-reactive protein with breast cancer, breast cancer subtypes, and poor outcome. Curr Probl Cancer. 2019;43(2):123-129.

15. Jiang $X$, Shapiro DJ. The immune system and inflammation in breast cancer. Mol Cell Endocrinol. 2014;382(1):673-682.

16. Hanahan D, Weinberg RA. Hallmarks of cancer: the next generation. Cell. 2011;144(5):646-74.

17. Qian BZ, Pollard JW. Macrophage diversity enhances tumor progression and metastasis. Cell. 2010;141(1):39-51.

18. Mantovani A. Molecular pathways linking inflammation and cancer. Curr Mol Med. 2010;10(4):369-73.

19. Hirano T. IL-6 in inflammation, autoimmunity and cancer. Int Immunol. 2021;33(3):127-148.

20. Ishihara K, Hirano T. IL-6 in autoimmune disease and chronic inflammatory proliferative disease. Cytokine Growth Factor Rev. 2002;13(4-5):357-68.

21. Murakami M, Harada M, Kamimura D, et al. Disease-association analysis of an inflammation-related feedback loop. Cell Rep. 2013;3(3):946-59.

22. Sergei G, Florian G, Michael K. Immunity, Inflammation, and Cancer. Cell. 2010;140(6):883-899.

23. Anand $P$, Kunnumakkara $A B$, Sundaram $C$, et al. Cancer is a preventable disease that requires major lifestyle changes. Pharm Res. 2008;25(9):2097-116.

24. Ishihara K, Sawa S, Ikushima $\mathrm{H}$, et al. The point mutation of tyrosine 759 of the IL-6 family cytokine receptor gp130 synergizes with HTLV-1 pX in promoting rheumatoid arthritis-like arthritis. Int Immunol. 2004;16(3):455-65.

25. Lippitz BE, Harris RA. Cytokine patterns in cancer patients: A review of the correlation between interleukin 6 and prognosis. Oncoimmunology. 2016;5(5):e1093722.

26. Hansen KC, D'Alessandro A, Clement CC, et al. Lymph formation, composition and circulation: a proteomics perspective. Int Immunol. 2015;27(5):219-27.

27. Veenstra TD, Conrads TP, Hood BL, et al. Biomarkers: mining the biofluid proteome. Mol Cell Proteomics. 2005;4(4):409-18.

28. Clement CC, Aphkhazava D, Nieves E, et al. Protein expression profiles of human lymph and plasma mapped by 2D-DIGE and 1D SDS-PAGE coupled with nanoLC-ESI-MS/MS bottom-up proteomics. J Proteomics. 2013;78:172-87.

29. Clement CC, Santambrogio L. The lymph self-antigen repertoire. Front Immunol. 2013;4:424.

30. D'Alessandro A, Dzieciatkowska M, Peltz ED, et al. Dynamic changes in rat mesenteric lymph proteins following trauma using label-free mass spectrometry. Shock. 2014;42(6):509-17.
31. Garnier L, Gkountidi AO, Hugues S. Tumor-Associated Lymphatic Vessel Features and Immunomodulatory Functions. Front Immunol. 2019;10:720.

32. Volk-Draper L, Patel R, Bhattarai N, et al. Myeloid-Derived Lymphatic Endothelial Cell Progenitors Significantly Contribute to Lymphatic Metastasis in Clinical Breast Cancer. Am J Pathol. 2019;189(11):2269-2292.

33. Osada M, Inoue O, Ding G, Shirai T, et al. Platelet activation receptor CLEC-2 regulates blood/lymphatic vessel separation by inhibiting proliferation, migration, and tube formation of lymphatic endothelial cells. J Biol Chem. 2012;287(26):22241-52.

34. Pan Y, Yago T, Fu J, et al. Podoplanin requires sialylated O-glycans for stable expression on lymphatic endothelial cells and for interaction with platelets. Blood. 2014;124(24):3656-65.

35. Welsh JD, Kahn ML, Sweet DT. Lymphovenous hemostasis and the role of platelets in regulating lymphatic flow and lymphatic vessel maturation. Blood. 2016;128(9):1169-73.

36. Tammela T, Alitalo K. Lymphangiogenesis: Molecular mechanisms and future promise. Cell. 2010;140(4):460-76.

37. Aleskandarany MA, Sonbul SN, Mukherjee A, et al. Molecular mechanisms underlying lymphovascular invasion in invasive breast cancer. Pathobiology. 2015;82(3-4):113-23.

38. Karkkainen MJ, Haiko P, Sainio K, et al. Vascular endothelial growth factor $\mathrm{C}$ is required for sprouting of the first lymphatic vessels from embryonic veins. Nat Immunol. 2004;5(1):74-80.

39. Foubert $P$, Varner JA. Integrins in tumor angiogenesis and lymphangiogenesis. Methods Mol Biol. 2012;757:471-86.

40. Cueni LN, Detmar M. The lymphatic system in health and disease. Lymphat Res Biol. 2008;6(3-4):109-22.

41. Adamczyk L.A., Gordon K, Kholová I, et al. Lymph vessels: the forgotten second circulation in health and disease. Virchows Arch.2016; 469:3-17.

42. Farnsworth RH, Achen MG, Stacker SA. The evolving role of lymphatics in cancer metastasis. Curr Opin Immunol. 2018;53:64-73.

43. Issa A, Le TX, Shoushtari AN, et al. Vascular endothelial growth factor-C and $\mathrm{C}-\mathrm{C}$ chemokine receptor 7 in tumor cell-lymphatic cross-talk promote invasive phenotype. Cancer Res. 2009; 69(1):349-57.

44. Shields JD, Fleury ME, Yong C, et al. Autologous chemotaxis as a mechanism of tumor cell homing to lymphatics via interstitial flow and autocrine CCR7 signaling. Cancer Cell. 2007;11(6):526-38.

45. Tutunea-Fatan E, Majumder M, Xin X, et al. The role of CCL21/CCR7 chemokine axis in breast cancer-induced lymphangiogenesis. $\mathrm{Mol}$ Cancer. 2015;14:35.

46. Chen Z, Varney ML, Backora MW, et al. Downregulation of vascular endothelial cell growth factor-C expression using small interfering RNA vectors in mammary tumors inhibits tumor lymphangiogenesis and spontaneous metastasis and enhances survival. Cancer Res. 2005;65:9004-9011.

47. Lin S, Kim J, Lee MJ, et al. Prospective transcriptomic pathway analysis of human lymphatic vascular insufficiency: identification and validation of a circulating biomarker panel. PLoS One. 2012;7(12):e52021. 